ARTICLE

https://doi.org/10.1038/s42003-019-0687-9 OPEN

\title{
Deconstructing and repurposing the light-regulated interplay between Arabidopsis phytochromes and interacting factors
}

\author{
David Golonka (1) ${ }^{1}$, Patrick Fischbach (1) ${ }^{2}$, Siddhartha G. Jena (10) ${ }^{3}$, Julius R.W. Kleeberg (1) ${ }^{1}$, Lars-Oliver Essen (1) ${ }^{4}$,

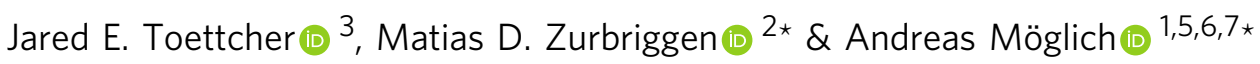

Phytochrome photoreceptors mediate adaptive responses of plants to red and far-red light. These responses generally entail light-regulated association between phytochromes and other proteins, among them the phytochrome-interacting factors (PIF). The interaction with Arabidopsis thaliana phytochrome B (AtPhyB) localizes to the bipartite APB motif of the $A$. thaliana PIFs (AtPIF). To address a dearth of quantitative interaction data, we construct and analyze numerous AtPIF3/6 variants. Red-light-activated binding is predominantly mediated by the APB N-terminus, whereas the $\mathrm{C}$-terminus modulates binding and underlies the differential affinity of AtPIF3 and AtPIF6. We identify AtPIF variants of reduced size, monomeric or homodimeric state, and with AtPhyB affinities between 10 and $700 \mathrm{nM}$. Optogenetically deployed in mammalian cells, the AtPIF variants drive light-regulated gene expression and membrane recruitment, in certain cases reducing basal activity and enhancing regulatory response. Moreover, our results provide hitherto unavailable quantitative insight into the AtPhyB:AtPIF interaction underpinning vital light-dependent responses in plants.

\footnotetext{
${ }^{1}$ Lehrstuhl für Biochemie, Universität Bayreuth, 95447 Bayreuth, Germany. ${ }^{2}$ Institute of Synthetic Biology and CEPLAS, Heinrich Heine University Düsseldorf, 40225 Düsseldorf, Germany. ${ }^{3}$ Department of Molecular Biology, Princeton University, Princeton, NJ 08544, USA. ${ }^{4}$ Department of Chemistry, Center for Synthetic Microbiology, Philipps University Marburg, 35032 Marburg, Germany. ${ }^{5}$ Research Center for Bio-Macromolecules, Universität Bayreuth, 95447 Bayreuth, Germany. ${ }^{6}$ Bayreuth Center for Biochemistry \& Molecular Biology, Universität Bayreuth, 95447 Bayreuth, Germany. ${ }^{7}$ North-Bavarian NMR Center, Universität Bayreuth, 95447 Bayreuth, Germany. ${ }^{*}$ email: matias.zurbriggen@uni-duesseldorf.de; andreas.moeglich@uni-bayreuth.de
} 
$\mathrm{F}$ irst discovered among the plant photoreceptors ${ }^{1}$, phytochromes (Phy) sense red and far-red light to control a range of physiological responses, including seedling germination, shade avoidance, entrainment of the circadian clock, and the transition from vegetative to reproductive growth ${ }^{2}$. Beyond plants, Phys also occur in bacteria and fungi where they mediate chromatic adaptation and pigmentation among other processes ${ }^{3,4}$. Receptors of the Phy family generally exhibit a bipartite architecture with an N-terminal photosensory core module (PCM) and a C-terminal output module (OPM) (Fig. 1a). The PCM of canonical Phys comprises consecutive PAS (Per/ARNT/Sim), GAF (cGMP-specific phosphodiesterase, adenylyl cyclase, and FhlA), and PHY (Phy-specific) domains and binds within its GAF domain a linear tetrapyrrole (bilin) chromophore ${ }^{3,5}$ (Fig. 1b). Phys of higher plants naturally employ phytochromobilin (РФВ), covalently attached to a cysteine residue within the GAF domain, but can be functionally reconstituted with phycocyanobilin (PCB) of cyanobacterial origin. In darkness, conventional Phys adopt their red-absorbing Pr state with the bilin chromophore in the $15 Z$ configuration; absorption of red light triggers rapid bilin isomerization to the $15 E$ state and population of the metastable, farred-absorbing Pfr state (Fig. 1b). The $\mathrm{Pfr} \rightarrow \mathrm{Pr}$ reversion occurs thermally or can be actively driven by far-red light. Insight from bacterial Phys illustrates that the $Z / E$ isomerization is coupled to refolding of the so-called PHY tongue, a protrusion of the PHY domain, from a $\beta$-hairpin to an $\alpha$-helix conformation, in turn prompting quaternary structural rearrangements ${ }^{6-10}$. Bacterial Phys mostly form part of two-component signaling cascades ${ }^{11}$ with OPMs acting as histidine kinases (HKs). By contrast, the Phy OPMs of land plants comprise two PAS domains, PAS-A and PAS-B, and a homologous HK-related domain that, however, lacks key residues essential for function and is thus devoid of HK activity. Rather, plant Phys have been reported to exhibit serine/threonine kinase activity ${ }^{12,13}$. Plant Phys exert their biological effects via light-regulated cytonucleoplasmic shuttling and protein:protein interactions (PPIs), which manifest in transcriptional responses and proteolytic degradation of cellular target proteins ${ }^{14-16}$. As one prominent protein family, the so-called phytochrome-interacting factors (PIFs) undergo light-regulated PPIs with plant Phys and act as basic helix-loop-helix transcription factors ${ }^{14,17-20}$ (Fig. 1c and Supplementary Fig. 1).

Arabidopsis thaliana possesses five Phys, denoted AtPhyA-E, that engage with a set of at least eight PIFs, denoted AtPIF1-8 ${ }^{14,17}$. For the arguably best-studied Phy, AtPhyB, preferential interactions of the Pfr state vs. the Pr state were identified with all eight AtPIFs ${ }^{14,17,21-23}$. Notably, the PCM of AtPhyB is necessary and sufficient for red-light-activated and farred-light-reversible AtPIF binding ${ }^{19,24-26}$. Although a pioneering study on the optogenetic use of $A t \mathrm{PhyB}$ reported that reversible interactions with AtPIF6 required the presence of PAS-A and $\mathrm{PAS} \mathrm{B}^{27}$, numerous later studies demonstrated that the PCM suffices for photoreversible interactions with AtPIFs ${ }^{28-30}$. That notwithstanding, the C-terminal OPM likely contributes to lightregulated PPIs and is integral to eliciting physiological responses ${ }^{14,15}$. Likewise, the light-activated interaction with AtPhyB maps to the weakly conserved APB (active phytochrome B binding) consensus motif within the $\mathrm{N}$-terminal region of AtPIF orthologs that precedes the basic helix-loop-helix domain ${ }^{24}$ (Fig. 1d). The APB motif consists of two segments, termed APB.A and APB.B, the first of which exhibits higher sequence conservation (Supplementary Fig. 1) and dominates light-activated AtPhyB binding as indicated by site-directed mutagenesis ${ }^{24}$. In the case of AtPhyA, the isolated PCM also suffices for light-regulated interactions with AtPIF1 and AtPIF3, which localize to the APA motifs (active phytochrome A binding) of these PIFs, somewhat C-terminal of the APB motifs ${ }^{31,32}$.
Early on, the light-regulated AtPhy:AtPIF PPI has been harnessed for the control of cellular processes in heterologous hosts by red and far-red light ${ }^{25,27}$, an approach now known as optogenetics $^{33}$. As manifold natural processes are intrinsically governed by PPIs, the AtPhy:AtPIF system provides a widely
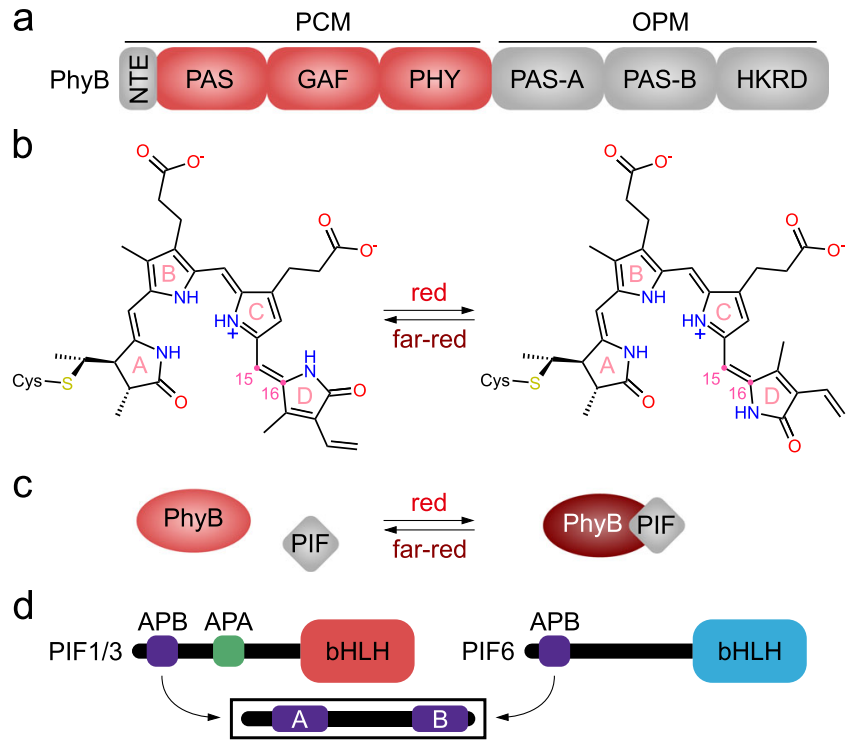

e

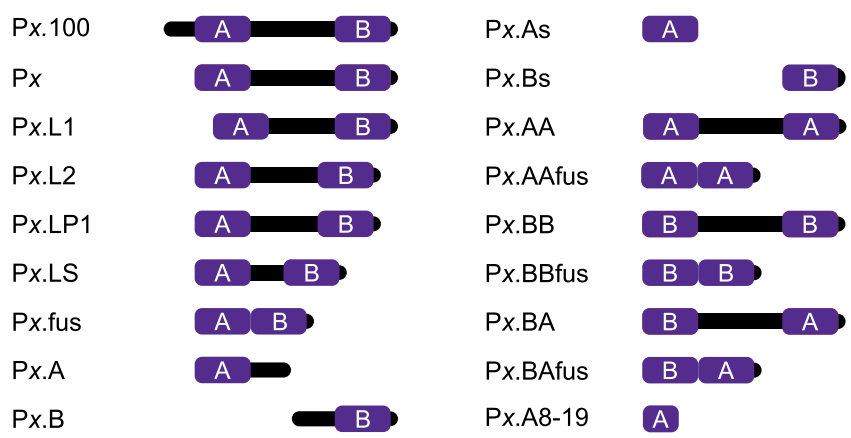

Fig. 1 Architecture and function of plant phytochromes (Phy) and their cognate phytochrome-interacting factors (PIFs). a Modular composition of plant phytochromes. An N-terminal extension (NTE) is succeeded by the photosensory core module (PCM) consisting of consecutive PAS, GAF, and PHY domains, with a phytochromobilin ( $P \Phi B$ ) chromophore covalently bound as a thioether within the GAF domain. The $C$-terminal output module (OPM) comprises two additional PAS domains (PAS-A and PAS-B), succeeded by a histidine-kinase-related domain (HKRD). $\mathbf{b}$ In the darkadapted $\operatorname{Pr}$ (red-absorbing) state of the Phy, the P $\Phi B$ chromophore adopts its $15 Z$ form. Red light drives isomerization to the $15 E$ form to give rise to the Pfr state (far-red-absorbing). Vice versa, far-red light drives the $\mathrm{Pfr} \rightarrow \mathrm{Pr}$ transition. c In their Pr state (red), plant Phys show no or at most weak interactions with PIFs. Following red-light absorption, the Pfr state (brown) is populated and affinity for the PIFs enhanced. $\mathbf{d}$ Modular composition of PIFs. An N-terminal region of around 100 residues contains the so-called $A P B$ motif that mediates interactions with phytochrome $B$. The APB motif further subdivides into the ABP.A and APB.B segments ${ }^{24}$. Certain PIFs also possess a more $C$-terminal APA motif engaged in interactions with phytochrome A. The C-terminal part comprises a basic helix-loop-helix (bHLH) DNA-binding domain. e Based on the N-terminal fragments of Arabidopsis thaliana PIFs 3 and 6, a panel of PIF variants were generated and probed for light-dependent protein:protein interactions with the PCM of $A$. thaliana PhyB (cf. Supplementary Table 1 for a detailed description of these derivatives). 
applicable means for the bimodal control of cellular phenomena with supreme resolution in space and time ${ }^{34}$. As a case in point, the expression of transgenes in yeast and mammalian cells has been subjected to red-/far-red-light control via a two-hybrid strategy ${ }^{25,35,36}$. To this end, a split transcription factor was engineered with one component of the AtPhy:AtPIF pair connected to a sequence-specific DNA-binding domain and the other to a transcriptional trans-activating domain. Exposure to red light prompts colocalization of the two entities and onset of expression from synthetic target promoters. In another approach ${ }^{27,37,38}$, the AtPhy:AtPIF pair conferred light sensitivity on plasma membrane recruitment and cellular signaling cascades in mammalian cells. Although details differ, optogenetic applications to date mostly employ the isolated PCM of AtPhyB and the N-terminal 100 amino acids of AtPIF3/6, denoted P3.100 and P6.100, that comprise the APB motif.

Despite the eminent role of the AtPhy:AtPIF interaction in nature and optogenetics, quantitative data on the interaction strength and the underlying sequence determinants are scarce. To fill this gap, we dissected and analyzed the light-dependent interaction between $A t \mathrm{PhyB}$ and $A t \mathrm{PIF} 3 / 6$ by several qualitative and quantitative approaches. Whereas the AtPhyB PCM bound P6.100 with about $10 \mathrm{nM}$ affinity in its Pfr state and showed no detectable affinity in the Pr state, P3.100 exhibited weaker Pfrstate affinity and elevated basal affinity in Pr. By deconstructing AtPIF3/6 and engineering a wide set of shortened variants, we pinpointed APB.A as decisive for light-regulated PPIs, with a modulatory role for APB.B. Quantitative analyses informed the construction of minimal AtPIF3/6 fragments of 25 and 23 residues, respectively, that retained stringently light-regulated PPIs with AtPhyB. When deployed for the optogenetic control of gene expression and membrane recruitment, the novel AtPIF variants with a range of interaction strengths achieved stratified and enhanced light responses.

\section{Results}

Deconstructing the AtPhyB:AtPIF interaction. Starting from the AtPIF constructs P3.100 and P6.100, we generated numerous derivatives with residues deleted from the $\mathrm{N}$ terminus, the linker between the APB.A and APB.B segments varied, or either segment omitted or duplicated (Fig. 1e, Supplementary Table 1). All AtPIF variants were C-terminally tagged with enhanced yellow fluorescent protein (EYFP) to promote protein solubility and facilitate concentration determination. We implemented a screening assay to efficiently probe interactions of these variants with the Pfr state of the AtPhyB PCM. The screen exploits the fact that AtPIF binding stabilizes the Pfr state of AtPhyB and decelerates the thermal reversion to the Pr state in the dark ${ }^{39}$ (Fig. 2a). For this assay, the AtPIF-EYFP variants were expressed in Escherichia coli, purified AtPhyB PCM was added to the crude cell lysate in substoichiometric amounts, and the $\mathrm{Pfr} \rightarrow \mathrm{Pr}$ reversion kinetics were monitored by absorption spectroscopy (Fig. 2b, c). The initial kinetics were normalized to an EYFPnegative control and provide a convenient readout for interactions (Fig. 2d). Although qualitative in nature, this first screening platform offers important advantages: (i) owing to the specificity of the AtPhyB:AtPIF interaction, the assay can be conducted in crude bacterial lysate, without the need for protein purification; and (ii) it can be easily multiplexed to test many variants in a single experiment.

A multiple sequence alignment of the $\mathrm{N}$-terminal regions of AtPIF1-8 delineates two regions of conservation that define the $A$ and B segments of the APB motif (Supplementary Fig. ${ }^{24}$ ). The APB.A segment shows stronger conservation and comprises a
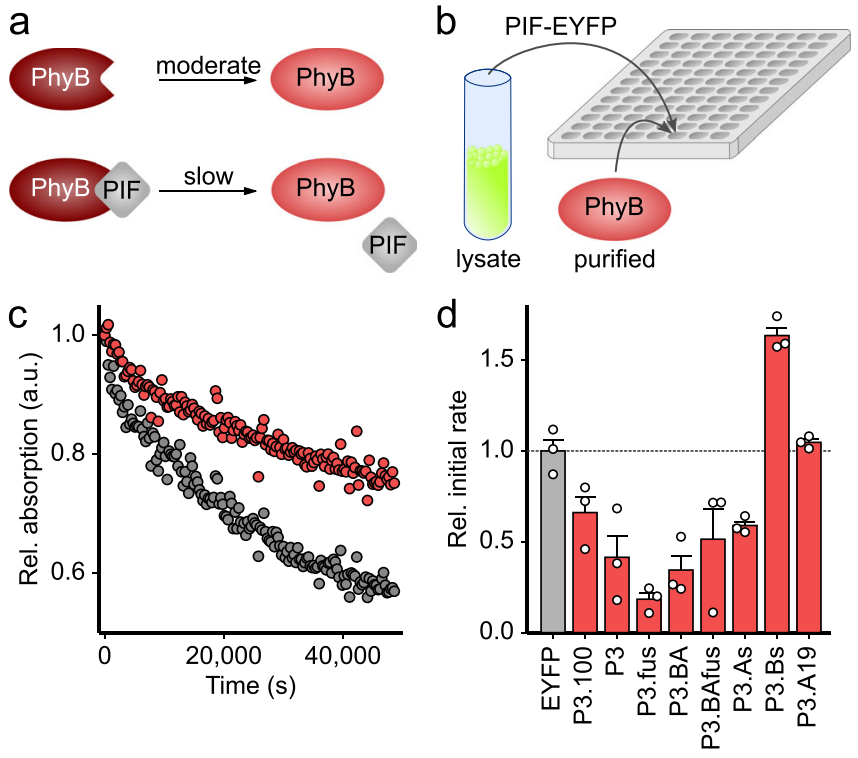

Fig. 2 Screening AtPIF variants for protein:protein interactions with the AtPhyB PCM. a The light-adapted Pfr state (brown) of AtPhyB thermally recovers to the dark-adapted $\operatorname{Pr}$ state (red) in a moderately paced reaction. When binding to an AtPIF variant, the recovery reaction is delayed. b AtPIF variants were C-terminally tagged with EYFP, expressed in Escherichia coli, cells were lysed, and AtPhyB PCM was added to the crude lysate. Samples were exposed to red light, and the recovery reaction was monitored over time by absorption measurements. c Normalized absorption of the AtPhyB PCM measured at $720 \mathrm{~nm}$ after red-light absorption in the presence of P3.100 (red) or the EYFP-negative control (gray). d The initial rates of the recovery reaction were determined and normalized to the reading obtained for the EYFP-negative control. Data indicate mean \pm SEM of $n=3$ independent biological replicates.

around 20 residues centered around the consensus core sequence ELXXXXGQ ${ }^{24}$; by comparison, the APB.B region is considerably shorter and less conserved. As the very $\mathrm{N}$-terminal region preceding APB.A varies substantially among the AtPIFs in length and sequence, we deemed it non-essential for AtPhyB interactions and removed it from $\mathrm{P} 3.100$ and $\mathrm{P} 6.100$. The resultant $\mathrm{P} x$ variants (here and in the following, $x=3,6$ ) retained interaction with the AtPhyB PCM, and all subsequent AtPIF variants were thus based on these N-terminally truncated forms (Fig. $2 \mathrm{~d}$ and Supplementary Fig. 2). Next, we interrogated the linkage between the constituent APB.A and APB.B segments, which is of heterogenous length and sequence across AtPIF1-8. We generated a set of variants, including (i) Px.L1 and Px.L2 in which the linkers of $\mathrm{P} 3 / \mathrm{P} 6$ are shortened by 10 residues at their $\mathrm{N}$ and $\mathrm{C}$ termini, respectively; (ii) Px.LP1 in which said linker is substituted for the corresponding segment of AtPIF1, the shortest among all AtPIFs; and (iii) Px.LS in which the linker is replaced by a repetitive glycine-serine stretch of 10 residues. As gauged by their effect on dark-reversion kinetics (cf. Supplementary Fig. 2), all these variants still interacted with the Pfr state of the AtPhyB PCM. These results imply that the linker connecting the APB.A and APB.B segments is dispensable, which is confirmed in the P $x$. fus variants that directly link these two segments without any linker and still exhibit interaction with the AtPhyB PCM (cf. Fig. 2d and Supplementary Fig. 2). To assess whether productive AtPhyB binding mandates a specific topology of the APB segments, we generated the variants $\mathrm{P} x . \mathrm{BA}$ and $\mathrm{P} x$.BAfus with 
inverted sequential order of APB.A and APB.B, and the original linker sequence kept or removed, respectively. Again, these variants retained interactions with the Pfr state of the AtPhyB PCM (cf. Fig. 2d and Supplementary Fig. 2). Site-directed mutagenesis had previously ascribed a dominant role to APB.A in mediating the light-dependent interaction with $A t \mathrm{PhyB}^{24}$, and we hence probed the two segments of the composite APB motif separately. Both the APB.A-containing variants $\mathrm{P} x$.A and the $\mathrm{P} x$. As, with or without the N-terminal half of the respective linker, still showed interactions with the AtPhyB PCM as judged by the effect on dark-reversion kinetics (cf. Fig. 2d and Supplementary Fig. 2). By contrast, neither the APB.B-based $\mathrm{P} x$.B nor the Px.Bs variants, with or without the C-terminal half of the linker, respectively, exhibited interactions in this assay. Duplication of the $\mathrm{A}$ part in the variants $\mathrm{P} x$.AA and $\mathrm{P} x$.AAfus preserved interactions with the AtPhyB PCM, and vice versa, duplication of the $\mathrm{B}$ segment in $\mathrm{P} x . \mathrm{BB}$ and $\mathrm{P} x$.BBfus failed to restore them (cf. Supplementary Fig. 2). Taken together, our findings emphasize the dominant role of APB.A for mediating interactions with AtPhyB. To further characterize the APB.A segment, we successively trimmed residues flanking its ELXXXXGQ core sequence. However, even the removal of five weakly conserved $\mathrm{C}$-terminal residues in the variants $\mathrm{P} x \mathrm{~A} 19$ abolished interactions with $A t P h y B$, as judged by their inability to slow down the AtPhyB-PCM $\mathrm{Pfr} \rightarrow \mathrm{Pr}$ reversion kinetics (cf. Fig. 2d and Supplementary Fig. 2). Likewise, no interaction with the AtPhyB PCM was detected for more extensive truncations of the APB.A segment (cf. Supplementary Fig. 2).

Biochemical analyses of the AtPhyB:AtPIF interaction. The above screening platform affords a qualitative first-pass assessment of the AtPIF variants but does not quantify the strength of interactions with $A t \mathrm{PhyB}$. Moreover, the assay is limited to interactions within the Pfr state but not the Pr state. We hence selected several of the above AtPIF candidates for in-depth analysis. Following expression and purification, we assessed the oligomeric state of these variants and of the AtPhyB PCM by sizeexclusion chromatography (SEC). In its $\mathrm{Pr}$ state, the isolated AtPhyB PCM elutes as a monomer with a minor homodimeric fraction, consistent with a recent SEC analysis ${ }^{40}$ (Fig. 3a). In the Pfr state, the predominantly monomeric state is maintained but the retention from the SEC column is slightly delayed, which arguably reflects light-induced conformational changes, i.e., a compaction, of the PCM that may resemble those observed in bacterial Phys ${ }^{6-8,10}$ (Fig. 3b). At a concentration of $10 \mu \mathrm{M}, \mathrm{P} 3.100$ and P6.100 largely eluted as homodimers with a minor monomeric population (Fig. 3c, Supplementary Fig. 3 and Table 1). Dimerization is not caused by the EYFP tag as the fluorescent protein itself eluted as a monomer (Fig. $3 \mathrm{~d}$, Table 1). Notably, the homodimeric state of AtPIFs is also observed in nature and critical for their physiological function as basic helix-loop-helix transcription factors ${ }^{41}$. Size reduction of the AtPIFs impaired homodimerization in several variants to different extent (Supplementary Fig. 3, Table 1). If the APB.A segment was truncated, as in P3.A and P6.A, or excluded altogether, as in P3.Bs and P6. $\mathrm{Bs}$, homodimerization was lost completely. Taken together, these findings point toward a contribution of the APB.A segment to homodimerization of the current AtPIF variants and, by extension, of the intact AtPIF3 and AtPIF6 proteins ${ }^{41}$.

We next investigated the interactions between the AtPIF3/6 variants and the AtPhyB PCM by SEC (Fig. 3e, Supplementary Fig. 4 and Table 1). To this end, we first converted the AtPhyB PCM to its Pfr state by illumination with red light $(640 \mathrm{~nm})$, incubated it at a 5:1 molar ratio with the different AtPIF variants,
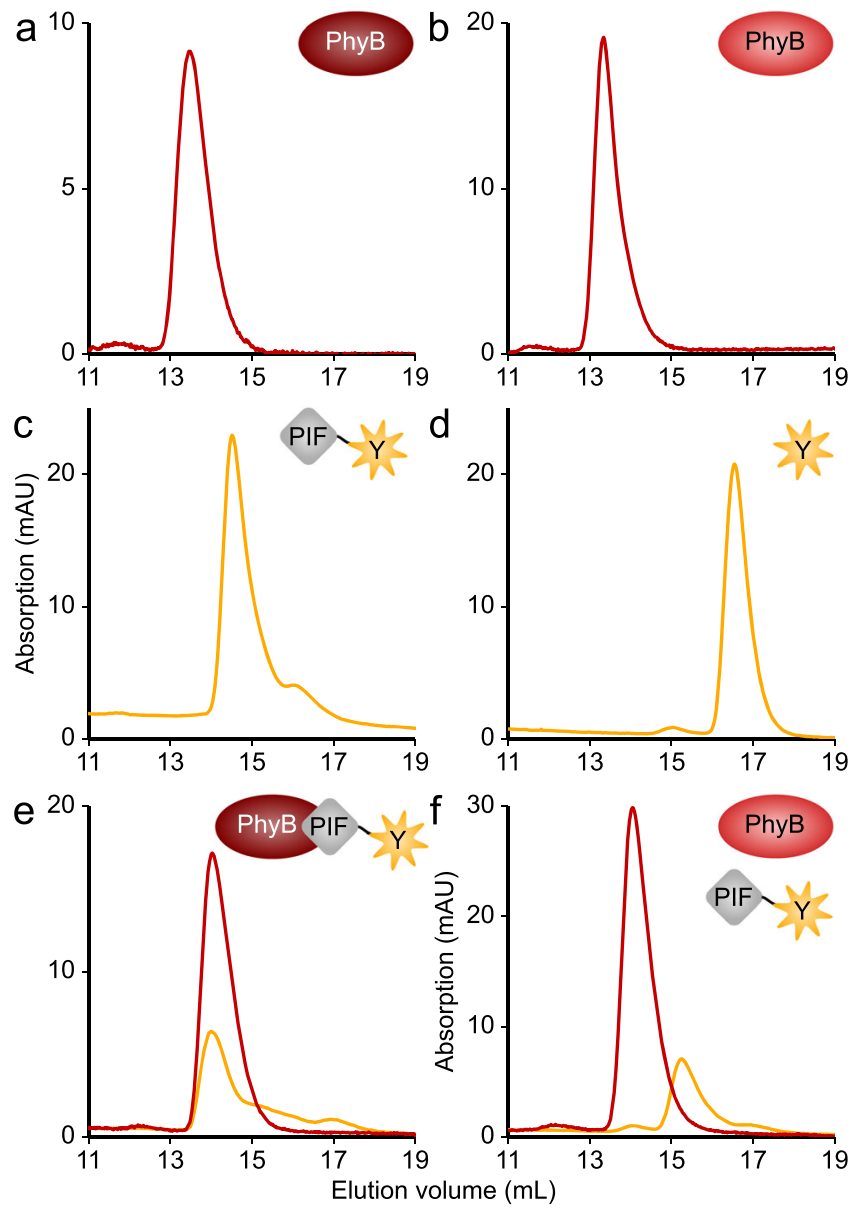

Fig. 3 Oligomeric state of the AtPIF variants and light-dependent interactions with the AtPhyB PCM. a $50 \mu \mathrm{M}$ AtPhyB PCM were exposed to red light and analyzed by size-exclusion chromatography (SEC), where the yellow and red lines represent absorption at 513 and $650 \mathrm{~nm}$, respectively. b As in a but the AtPhyB PCM was exposed to far-red light prior to chromatography. c $10 \mu \mathrm{M}$ P3.100-EYFP were analyzed by SEC. Elution profiles were independent of illumination. d $10 \mu \mathrm{M}$ of the negative control EYFP were analyzed by SEC. Elution profiles were independent of light. e A mixture of $10 \mu \mathrm{M}$ P3.100-EYFP and $50 \mu \mathrm{M}$ AtPhyB PCM was exposed to red light and analyzed by SEC. $\mathbf{f}$ As in e but samples were illuminated with far-red light, rather than red light. Experiments were repeated twice with similar results.

and analyzed the mixture by SEC. In full agreement with the firstpass screening (cf. Fig. 2 and Supplementary Fig. 2), all variants that we had identified as binding-competent exhibited interactions with AtPhyB PCM at an apparent 1:1 stoichiometry. Vice versa, the AtPIF variants that had failed to decelerate AtPhyB reversion kinetics (cf. Fig. 2 and Supplementary Fig. 2) lacked any interactions (Supplementary Fig. 4). We also assessed interactions between the AtPIF variants and the AtPhyB PCM in the Pr state following exposure to far-red light $(720 \mathrm{~nm}$ ) (Fig. $3 \mathrm{f}$ and Supplementary Fig. 5). None of the variants showed interactions under these conditions. Insofar red-light-activated binding to the AtPhyB PCM had been retained in the truncated AtPIF variants, far-red light hence abolished it.

Having engineered a suite of AtPIF variants undergoing lightregulated PPIs with the AtPhyB PCM, we next sought to quantify the strength of these interaction in both the Pr and Pfr states. 
Table 1 Biochemical analyses of the AtPIF3/6 variants.

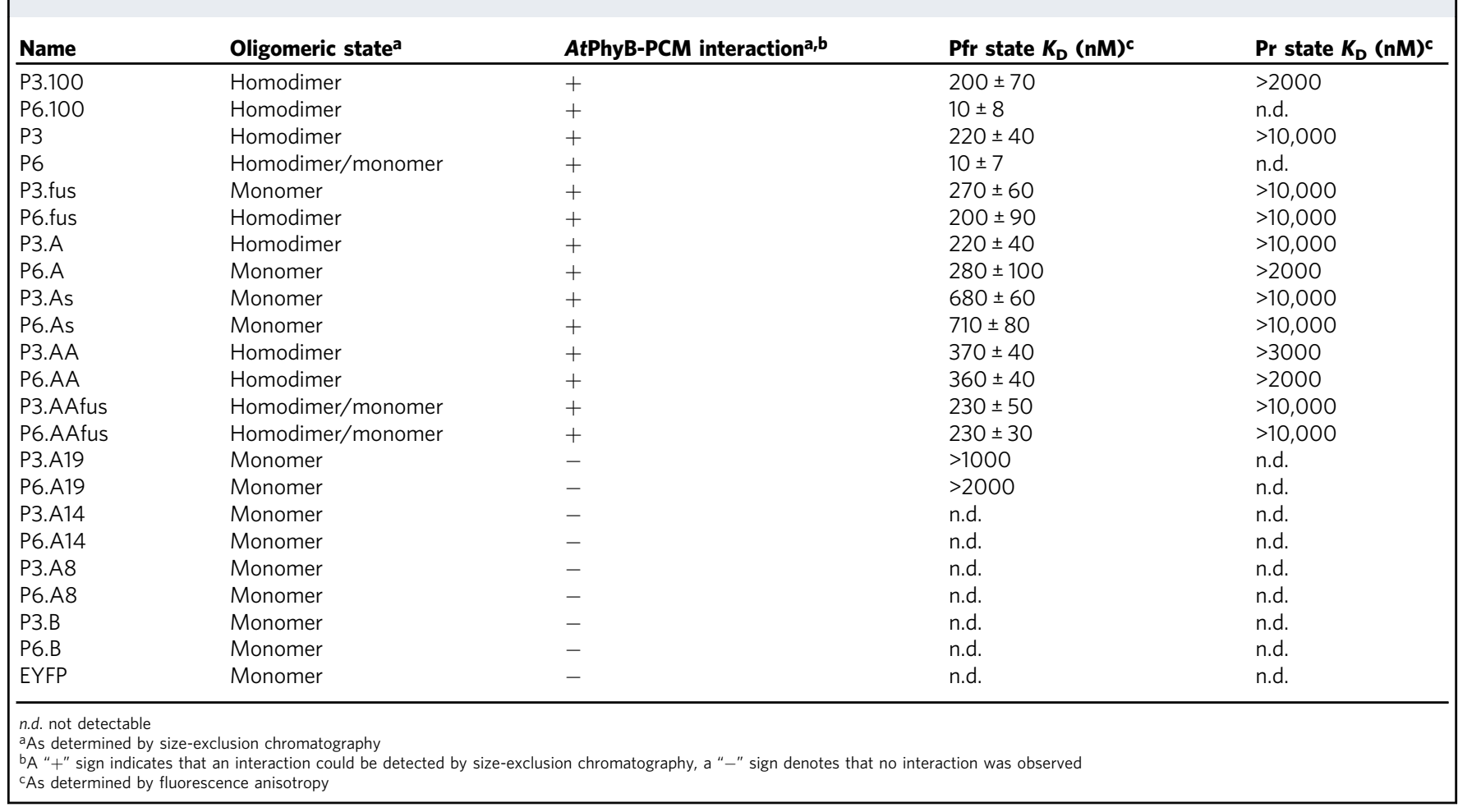

Notably, detailed quantitative data of that type are largely unavailable but would tremendously improve our understanding of the AtPhyB:AtPIF PPI and inform its optimization. To this end, we resorted to fluorescence anisotropy measurements of the EYFP moiety C-terminally appended to all the AtPIF variants. Binding of a given AtPIF-EYFP variant to the AtPhyB PCM would increase its effective hydrodynamic radius, slow down rotational diffusion, and thus increase fluorescence anisotropy (Fig. 4a). We hence incubated a constant $20 \mathrm{nM}$ of the AtPIF-EYFP variants with increasing amounts of AtPhyB PCM under red or far-red light and recorded binding isotherms. The reference construct P6.100 exhibited strong binding to the AtPhyB PCM under red light but no detectable binding under far-red light even at AtPhyB-PCM concentrations of $2 \mu \mathrm{M}$ (Fig. $4 \mathrm{~b}$ ). When calculating dissociation constants $\left(K_{\mathrm{D}}\right)$, one must consider that red light not only drives the $\mathrm{Pr} \rightarrow \mathrm{Pfr}$ transition of Phys but also the reverse $\mathrm{Pfr} \rightarrow \operatorname{Pr}$ process. Consequently, continuous illumination with red light $(640 \mathrm{~nm})$ leads to population of a photostationary state with a mixed $\mathrm{Pfr} / \mathrm{Pr}$ population at a ratio of $\sim 0.56 / 0.44^{42}$ (Fig. 4c). Correcting for the actual fraction in the Pfr state, we determined a $K_{\mathrm{D}}$ for the P6.100:AtPhyB-PCM pair of $10 \pm$ $8 \mathrm{nM}$ (Table 1). This value is in good agreement with an earlier estimate for this pair of 20-100 nM within mammalian cells based on fluorescence microscopy ${ }^{27}$. In comparison to P6.100, P3.100 exhibited a weaker $K_{\mathrm{D}}$ of $200 \pm 70 \mathrm{nM}$ in Pfr and an elevated basal affinity in Pr, with an estimated $K_{\mathrm{D}}$ on the order of low micromolar (Fig. 4d and Table 1). This residual interaction could in principle be due to partial population of the Pfr state of the AtPhyB PCM under the chosen illumination conditions; however, the absence of basal affinity in case of P6.100 strongly argues against this notion. The slightly weaker affinity and much less pronounced light effect in P3.100 compared to P6.100 may account for the previously reported inability to detect light-regulated interactions of AtPIF3 with the AtPhyB PCM in mammalian cells ${ }^{27}$. We then recorded binding isotherms under red and far-red light for all the AtPIF variants we had purified and analyzed by SEC (Supplementary Figs. 4 and 5, Table 1). Consistent with our first-pass assessment (cf. Fig. 2d and Supplementary Fig. 2), the removal of the nonconserved N-terminal residues preceding the APB.A segment had no influence on the Pfr interaction. Unexpectedly, omission of these residues in the AtPIF3 context substantially attenuated the basal Prstate affinity. For the AtPIF3 variants, removal of the linker and the APB.B part had no or at most modest effects on affinity to the Pfr state (Supplementary Fig. 6, Table 1). By contrast, in AtPIF6, the removal of the linker and the APB.B part more severely attenuated the affinity to the Pfr state to values between 200 and $700 \mathrm{nM}$. In addition, the affinity to the Pr state, non-detectable for the variants P6.100 and P6, increased as well. As a corollary, AtPIF3 and AtPIF6 variants lacking the APB.B segment exhibited closely similar $K_{\mathrm{D}}$ values for a given construct topology. As a case in point, the P3.As and the P6.As variants, comprising 25 and 23 residues, respectively, both interacted with the AtPhyB PCM with an affinity of $\sim 700 \mathrm{nM}$ in the Pfr and weaker than $10 \mu \mathrm{M}$ in the Pr state. These data for P6As are consistent with a recent report that demonstrated lightdependent PPI for an AtPIF6 construct of closely similar length and sequence $^{43}$. Duplication of the APB.A segments in the AtPIF3/6 backgrounds resulted in variants with affinities in the range of $200-400 \mathrm{nM}$ for Pfr and weaker than $2 \mu \mathrm{M}$ for Pr. We also analyzed several AtPIF3/6 variants entirely lacking the APB.A segment or possessing shortened versions of it, neither of which showed any interaction with AtPhyB PCM when probed by SEC nor by their effect on $\operatorname{Pr}$ reversion kinetics. In almost all these variants, fluorescence anisotropy failed to detect interactions either (Supplementary Fig. 6 and Table 1); merely, the P3.A19 and P6.A19 variants with C-terminally trimmed APB.A segments exhibited weak affinity for the Pfr state in the low micromolar range (Supplementary Fig. 6 and Table 1). In summary, these results 

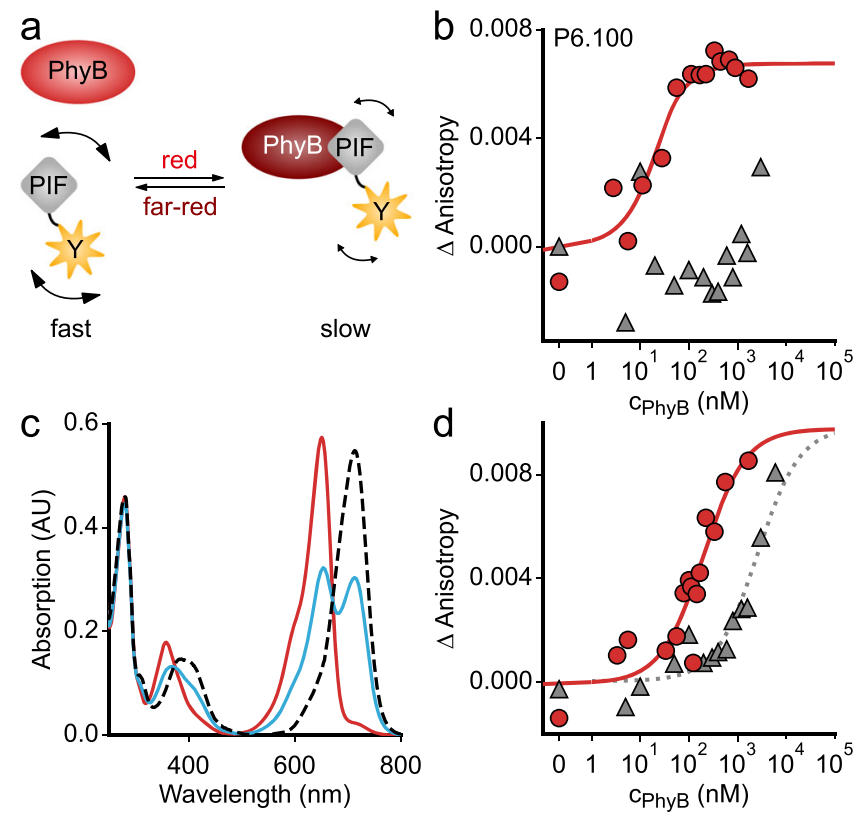

Fig. 4 Quantitative analyses of the light-dependent protein:protein interaction between AtPIF variants and the AtPhyB PCM. a In its Pr state, the AtPhyB PCM exhibits weak or no affinity to AtPIF, but upon red-light exposure, the affinity is enhanced. Binding to the AtPhyB PCM increases the effective hydrodynamic radius of the AtPIF variants and slows down rotational diffusion. In turn, the fluorescence anisotropy of an EYFP tag C-terminally appended to the AtPIF increases. b Titration of 20 nM P6.100EYFP with increasing concentrations of dark-adapted (gray) or red-lightexposed AtPhyB PCM (red), as monitored by anisotropy of the EYFP fluorescence. Data points show mean of $n=3$ biological replicates. The red line denotes a fit to a single-site-binding isotherm. $\mathbf{c}$ Absorption spectra of the AtPhyB PCM in its dark-adapted Pr state (red line) and as a Pfr/Pr mixture following red-light exposure (blue). The dashed line denotes the absorption spectrum of the pure Pfr state, calculated according to ref. 42 d As in $\mathbf{b}$ but for P3.100-EYFP rather than P6.100-EYFP. Experiments were repeated twice with similar results.

confirm the APB.A segment as the main interaction epitope in both AtPIF3 and AtPIF6. Intriguingly, AtPIF6 differs from AtPIF3 by higher affinity for Pfr and much reduced affinity for Pr. As the removal of the APB.B segment largely cancels these differences, we conclude that APB.B in AtPIF6, but not in AtPIF3, enhances the affinity for Pfr and diminishes that for Pr. In AtPIF3, the Nterminal amino acids contribute to elevated basal affinity for Pr.

Repurposing the AtPhyB:AtPIF interaction for optogenetics. Through sequence variations and quantitative analyses, we generated modules for light-regulated PPIs spanning an affinity for the Pfr state from around 10 to $700 \mathrm{nM}$. We next investigated whether this set of novel AtPIF variants can be leveraged for optogenetics in mammalian cells. In a first line of experiments, we embedded the variants into a previously reported system for red-/ far-red-light-regulated gene expression that provides an in-cell readout of relative PPI affinities ${ }^{36,44}$. To this end, the AtPhyB PCM was covalently attached to a VP16 trans-activating domain, and the different AtPIF variants were linked to the E-protein DNA-binding domain, which binds to a cognate operator sequence upstream of a minimal promoter driving expression of secreted alkaline phosphatase (SEAP) (Fig. 5a). Through light-induced AtPhyB:AtPIF interactions, the trans-activating domain localizes to the DNA-binding domain and the promoter and thereby induces SEAP expression. SEAP activity levels are quantified and normalized to the levels of constitutively expressed Gaussia luciferase to correct for variations of cell density, transfection efficiency, and overall expression. We found that the P3.100 and P6.100 reference constructs upregulated normalized SEAP expression by tenfold and fourfold, respectively, under red light compared to darkness when expressed in Chinese hamster ovary cells (CHO-K1). The comparatively small regulatory effect for P6.100 results from substantial basal SEAP expression. We then subjected all the AtPIF3/6 variants we had previously characterized to the same analysis (Fig. 5b, c and Supplementary Fig. 7). Consistent with the above measurements, AtPIF variants that lacked detectable interactions with the AtPhyB PCM, e.g., P $x$. $\mathrm{B}$ and $\mathrm{P} x . \mathrm{A} 19$, failed to stimulate reporter expression regardless of illumination. By contrast, variants that exhibited interactions with the AtPhyB PCM were generally capable of inducing SEAP expression under red light, albeit to different degree. Overall, the expression levels observed for the individual AtPIF variants scaled with binding affinity, in that low measured $K_{\mathrm{D}}$ values correlated with strong SEAP activity. For instance, all AtPIF3/6 variants containing the intact APB.A segment exhibited strong expression under red light. Whereas P6.100 suffered from relatively high basal expression, the shortened AtPIF6 derivatives generally showed reduced SEAP expression in darkness, translating into much more pronounced regulatory effects. For instance, in the variant P6.A the SEAP expression increased by 43 -fold under red light relative to darkness. Duplication of APB.A in the variants P6.AA and P3.AA elevated SEAP expression under red light and, to lesser extent, in darkness, thereby enhancing the regulatory effect. The overall higher SEAP expression under red light for these variants could reflect the binding of two AtPhyB-VP16 modules to one $\mathrm{P} x$.AA protein. However, we note that, under the conditions employed for the SEC analysis, we did not find evidence for simultaneous binding of two AtPhyB PCM entities to the Px.AA variants. We also assessed the photoreversibility of the gene-expression systems based on the AtPIF derivatives (Supplementary Fig. 8). When the cells were first exposed to red light for $24 \mathrm{~h}$, followed by far-red illumination for another $24 \mathrm{~h}$, they exhibited basal SEAP expression levels comparable to cells incubated in darkness throughout. Given that gene expression for the different sequence variations followed similar trends in both the AtPIF3 and the AtPIF6 backgrounds, we wondered whether the emerging underlying principles extend to other AtPIF orthologs. We hence generated the corresponding sequence variations in the AtPIF1 background and assessed their impact on light-regulated gene expression (Fig. 5d and Supplementary Fig. 7). Several of the resultant AtPIF1 variants supported lightactivated SEAP expression, although generally with slightly attenuated maximal levels and regulatory effects. Nonetheless, the AtPIF1 variants conformed to the general activity pattern observed for the AtPIF3/6 variants; specifically, only the AtPIF1 variants preserving an intact APB.A segment were capable of upregulating SEAP expression under red light. Taken together, these experiments demonstrate the utility of the cellular set-up for the efficient appraisal of light-regulated PPIs in mammalian cells. By capitalizing on this set-up, we obtained derivative systems with enhanced dynamic range and reduced leakiness that outperformed the original reference systems.

In a second set of experiments, we deployed several of the newly generated AtPIF6 variants for light-regulated recruitment of target proteins to the plasma membrane of NIH-3T3 cells. To this end, we equipped the AtPhyB PCM with a C-terminal CAAX prenylation motif for membrane targeting and the AtPIF6 


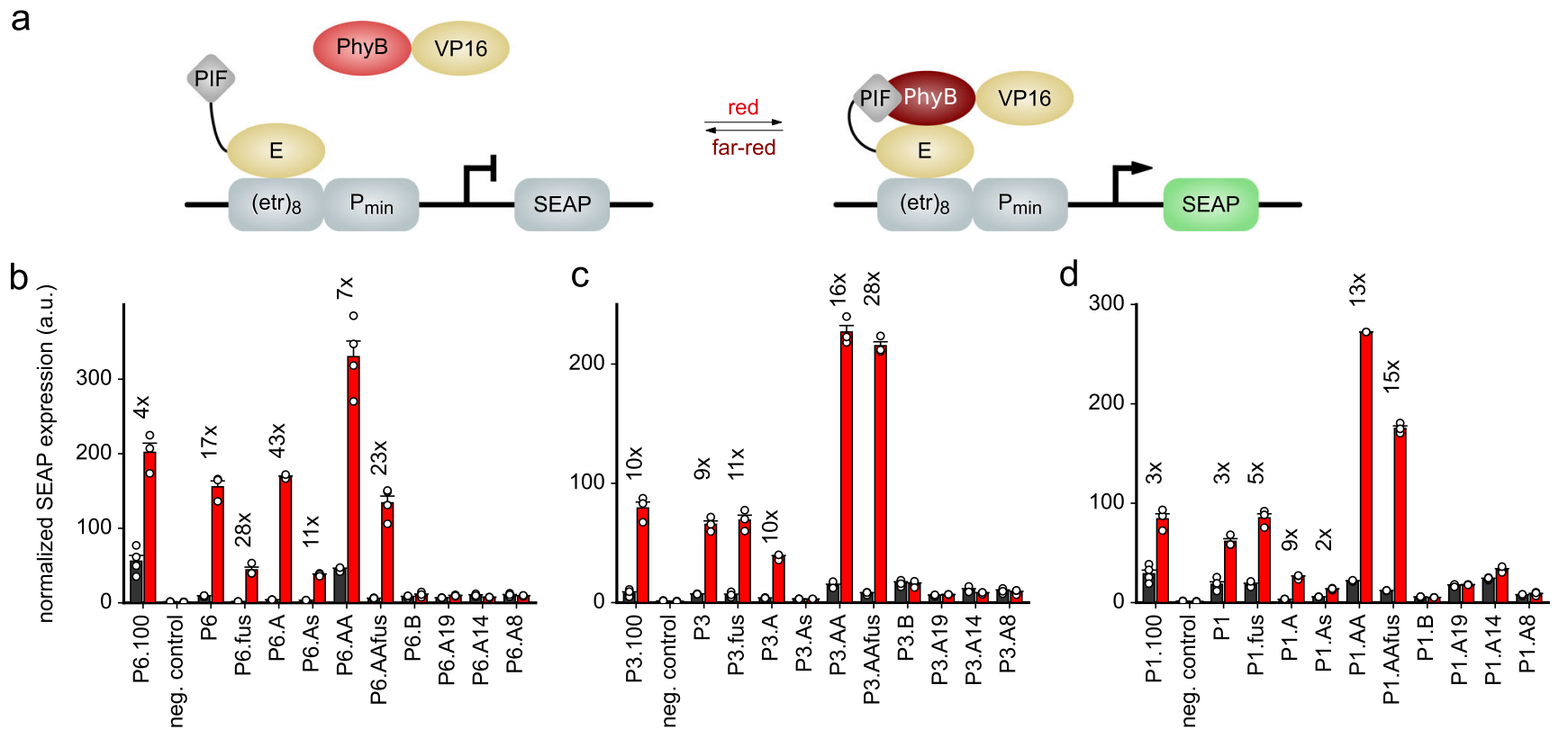

Fig. 5 Harnessing the AtPIF variants for the light-dependent regulation of gene expression in mammalian cells. a The AtPhyB PCM and AtPIF variants are connected to a VP16 trans-activating domain and an E-protein DNA-binding domain that binds to a synthetic promoter sequence. Red light promotes association of the AtPhyB:AtPIF pair and thereby activates the expression of a secreted alkaline phosphatase (SEAP) reporter gene. b SEAP expression was determined in Chinese hamster ovary cells ( $\mathrm{CHO}-\mathrm{K} 1$ ) for the diverse AtPIF6 variants and normalized to the constitutive expression of Gaussia luciferase. Black and red bars denote mean \pm SEM normalized SEAP expression for $n=4$ independent biological replicates under dark or red-light conditions, respectively. Cells were kept in darkness for $24 \mathrm{~h}$, supplemented with PCB, and then either kept in darkness for $24 \mathrm{~h}$ or illuminated for $24 \mathrm{~h}$ with $20 \mu \mathrm{mol} \mathrm{m} \mathrm{m}^{-2} \mathrm{~s}^{-1} 660-\mathrm{nm}$ light. As a negative control, the reporter construct alone was transfected. The numbers above the bars indicate the factor difference between dark and red-light conditions for a given AtPIF6 variant. c As b but for the AtPIF3 variants. d As $\mathbf{b}$ but for the AtPIF1 variants.

variants with an N-terminal EYFP tag 27,37,38 (Fig. 6a). Cell lines stably expressing both the AtPhyB PCM and one of the AtPIF6 variants, linked by an internal ribosome entry site (IRES), were created through lentiviral transduction. Cells were exposed to red $(650 \mathrm{~nm})$ and far-red light $(750 \mathrm{~nm})$, respectively, and the subcellular distribution of the EYFP-AtPIF6 variants was monitored by fluorescence microscopy (Fig. 6b-e). Under farred light, the reference variant P6.100 mostly localized to the cytoplasm, but under red light it partially translocated to the plasma membrane (Fig. 6c-f). Whereas the variants P6.A, P6.As, and P6.AA exhibited overall similar subcellular distribution under red and far-red light as P6.100, the variant P6.fus failed to show any light response of subcellular localization. Although subtle performance differences between the individual AtPIF6 variants cannot be ruled out, these are exceeded by the cell-to-cell variability of light-dependent translocation (Fig. 6f). Nonetheless, the experiments show that the new AtPIF6 variants with a much smaller footprint support light-regulated plasma membrane recruitment at similar efficiencies as the reference P6.100. This notion is further supported by the overall comparable expression level of the AtPIF6 variants and its effect on the magnitude of light-regulated membrane recruitment (Fig. 6g).

\section{Discussion}

In this study, we have dissected the light-regulated PPIs between the AtPhyB PCM and the AtPIFs 3 and 6, which underpin diverse adaptive responses in planta and multiple applications in optogenetics. To this end, we implemented a set of complementary experimental approaches ranging from SEC and fluorescence anisotropy to reporter assays in mammalian cells that deliver both qualitative and quantitative information on the PPIs. At a qualitative level, these assays consistently showed the APB.A segment to be necessary and sufficient for AtPhyB-PCM interactions, in line with previous reports ${ }^{24}$. By contrast, the APB.B segment alone did not promote detectable interactions. Our quantitative analyses put concrete numbers on the affinity of the AtPhyB:AtPIF3/6 pairs, information that hitherto was largely lacking. Strikingly, P6.100 exhibited a $K_{\mathrm{D}}$ of only $\sim 10 \mathrm{nM}$ for AtPhyB PCM in its Pfr state but entirely lacked interaction with the Pr state, from which we estimate an at least 1000-fold affinity difference. By contrast, the light dependence of the P3.100: AtPhyB-PCM interaction was less pronounced, with dissociation constants of $\sim 200 \mathrm{nM}$ in the Pfr state and low micromolar in the Pr state. We tied the more stringent red-light response in AtPIF6 to its APB.B segment, which enhances affinity for the Pfr state of the AtPhyB PCM while simultaneously attenuating basal affinity for the Pr state. We speculate that these inherent differences between AtPIF3 and AtPIF6 might reflect their natural roles in planta. Whereas AtPIF3 predominantly serves as a negative regulator of photomorphogenesis by modulating the abundance of $A t \mathrm{PhyB}^{45-47}$, AtPIF6 acts as a positive regulator by inhibiting hypocotyl elongation under red light, at least when overexpressed ${ }^{48}$. To prevent untimely inhibition of hypocotyl growth, a more stringent light response with very low basal affinity in $\mathrm{Pr}$ may be required for this particular PIF. Recently, it has been reported that PIFs, and in particular AtPIF3, are constantly turned over both in darkness and under red light as a mechanism of achieving optimal levels for tight regulation of the skotomorphogenic and photomorphogenic responses ${ }^{14}$. A more permissive binding of AtPIF3 to the Pr state of AtPhyB as observed here 
a

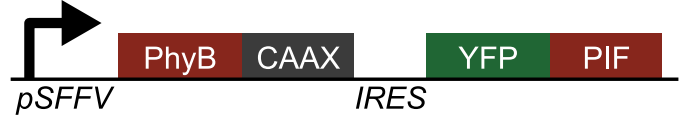

b

membrane

Phy-CAAX

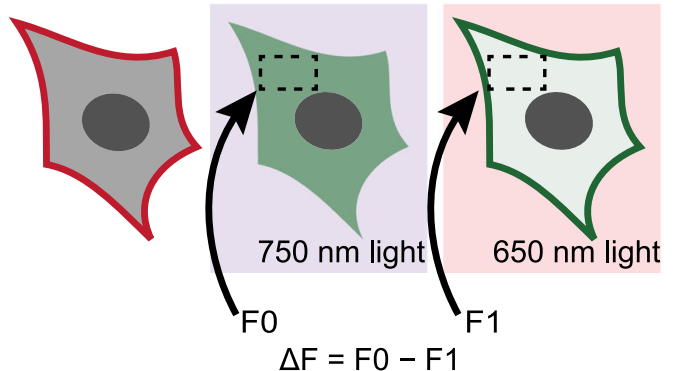

C

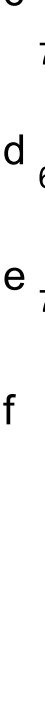

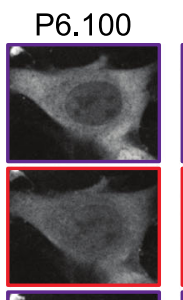
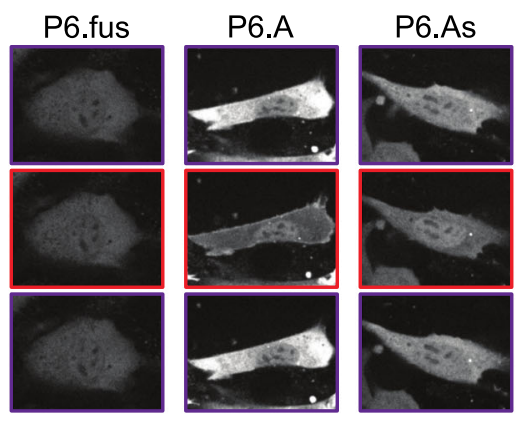

P6.AA

g

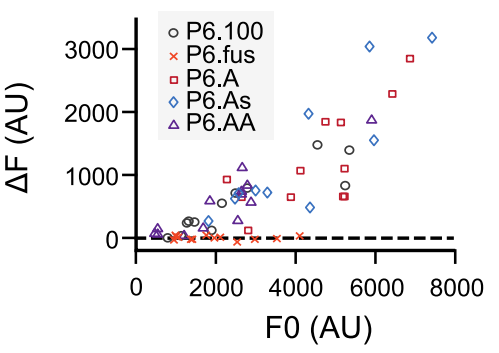

Fig. 6 Photoreversible recruitment to the plasma membrane. a AtPhyB and one of the several new AtPIF6 variants, equipped with a C-terminal CAAX prenylation motif or an N-terminal EYFP tag, respectively, were encoded on a bicistronic vector with an intervening IRES sequence and expressed in NIH3 T3 cells. b Owing to the CAAX tag, AtPhyB localizes to the plasma membrane, while the EYFP-AtPIF6 variants shuttle between cytosol and plasma

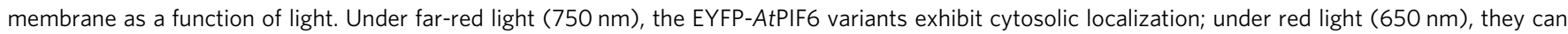
bind to AtPhyB and translocate to the membrane. c-e Fluorescence micrographs of NIH-3T3 cells expressing AtPhyB-CAAX and different EYFP-AtPIF6 variants under far-red light (c), after red-light exposure (d), and after additional exposure to far-red light (e). The scale bar denotes $20 \mu \mathrm{m}$. $\mathbf{f}$ The relative depletion of cytosolic EYFP fluorescence under red light compared to far-red light for the EYFP-AtPIF6 variants. Data represent mean \pm SD of $n \geq 12$ individual cells. $\mathbf{g}$ Dependence of the relative fluorescence change on the overall EYFP-AtPIF6 expression level.

might facilitate the regulation of PIF abundance in darkness. This concurs with reports that AtPhyB mediates phosphorylation by PPK-family kinases and subsequent degradation of AtPIF3 in both the Pr and Pfr states ${ }^{49}$. The differential affinities of the individual PIFs might therefore contribute to the fine-tuning of physiological responses ${ }^{14,49}$. In fact, our study now provides a means of gradually adjusting the interaction strength of a given PIF, which could benefit the analysis of signal transduction mechanisms in planta. In a similar vein, the quantitative data on the AtPhyB:AtPIF PPI may help rationalize the phenotypes of pertinent pif mutant alleles. Finally, the comparatively smaller regulatory effect in AtPhyB:AtPIF3, compared to AtPhyB:AtPIF6, may explain why this PPI pair proved inferior for generic optogenetic applications ${ }^{27}$.

By deconstructing and quantitatively analyzing AtPIF3/6, we devised a suite of interaction modules with several beneficial traits (Table 1): First, the AtPIF variants span an affinity range from 10 to $700 \mathrm{nM}$, thus enabling the precise tuning of light-regulated PPIs as demanded by a specific application. Second, the AtPIFs can be reduced to around $23-25$ residues while largely retaining lightregulated PPIs with the AtPhyB PCM. As we demonstrate, the smaller size facilitates the construction of tandem repeats of the APB.A motif, which, depending upon context, may enhance lightdependent responses. Third, the reduction in size also affected the oligomeric state of the AtPIFs, which are homodimeric at full length ${ }^{41}$ but predominantly monomeric in several of the truncated variants studied presently. As we showcase for the scenarios of light-regulated gene expression and membrane recruitment, the set of novel AtPIF variants can indeed improve absolute activity and degree of light regulation in optogenetics. As a case in point, despite stringently light-regulated PPIs with the AtPhyB PCM, the original P6.100 variant promoted substantial basal gene expression in darkness, thus degrading the regulatory effect of light. We tentatively ascribe the relatively poor performance of P6.100 to its high Pfr-state affinity; even limited population of the AtPhyB Pfr state, e.g., due to light pollution or temperature changes ${ }^{50}$, may hence activate the PPI to considerable extent and over prolonged periods ${ }^{39}$. In support of this notion, the attenuation of the Pfr-state affinity in the shortened AtPIF6 variants led to reduced basal activity and enhanced regulatory efficiency. Duplication of the APB.A segment improved the performance for light-regulated expression, although the Pfr-state affinity of the P $x$.AA variants is almost unchanged relative to the corresponding $\mathrm{P} x$.A variants. We hence ascribe this improvement to avidity and cooperativity effects. Our analyses readily extended to the AtPIF1 context, where shortened variants exhibited similar patterns of activity and light regulation as the AtPIF3/6 variants (cf. Fig. 5b-d). We speculate that the underlying principles can be generalized to APBcontaining PIF proteins from A. thaliana and other plants $\mathrm{s}^{51,52}$ The performance of individual AtPIF variants in a given experiment can considerably vary and may be difficult to gauge upfront, not least because it likely depends on application context. We thus consider it an advantage to have now a set of AtPIF variants with known interaction strengths and varying properties. With this suite of AtPIF variants in hand, additional processes may be unlocked for optogenetic control by red and far-red light. As recently summarized ${ }^{53}$, numerous cellular parameters and pathways depend on PPIs and can thus be controlled by certain photoreceptors that associate or dissociate under blue light. The underlying regulatory strategy should readily extend to the present AtPhyB:AtPIF pairs and thereby to red and far-red light. Other potential use cases for the new AtPIF variants include immunoreceptor signaling ${ }^{30}$ and light-regulated biomaterials ${ }^{54}$. As one shortcoming, optogenetic applications of plant Phys currently require the exogenous addition of $\mathrm{PCB}$ or $\mathrm{P} Ф \mathrm{~B}$ chromophores, which do not widely occur outside cyanobacteria and plants. This contrasts with bacterial Phys, which utilize biliverdin (BV) that is available in mammals as a heme degradation 
product ${ }^{55-58}$. In particular, a recently described bacterial Phy undergoes PPIs depending on red and far-red light and has been harnessed for light-regulated gene expression ${ }^{59-61}$. The reliance on $\mathrm{BV}$ in this system obviates exogenous chromophore addition, which may prove advantageous for applications in vivo.

In summary, we have constructed and characterized a toolkit of novel AtPIF variants with varying interaction strength, size, and oligomeric state. Beyond application in optogenetics, the availability of these variants also stands to benefit the biophysical analyses of the Phy:PIF interaction. Although previous studies had localized this interaction to the $\mathrm{N}$-terminal extension of Phys, atomically resolved information on the Phy:PIF complex is lacking 40,62-64. Minimized AtPIFs may well facilitate X-ray crystallographic analysis and thus pave the way toward elucidation of the complex structure. Moreover, the qualitative and quantitative interaction assays presently established can be deployed to chart Phys and interacting factors from A. thaliana and other plants.

\section{Methods}

Molecular biology and protein purification. Genes encoding A. thaliana PhyB PCM (residues 1-651), PIF3 (1-100), and PIF6 (1-100) were synthesized with codon usage adapted for expression in E. coli (GeneArt, Invitrogen, Regensburg, Germany). Via Gibson assembly ${ }^{65}$, the AtPhyB PCM was furnished with a Cterminal hexahistidine tag and subcloned onto the pCDFDuet1 vector (Novagen, Merck, Darmstadt, Germany) under control of a T7-lacO promoter; the plasmid, designated pDG282, additionally harbors a bicistronic cassette of Synechocystis sp. heme oxygenase 1 and $\mathrm{pcyA}^{66}$, also under the control of T7-lacO. For the expression of AtPIF3/6, the corresponding genes were subcloned onto a pET-19b vector (Novagen) under the control of a T7-lacO promoter by Gibson assembly or AQUA cloning ${ }^{67}$ and thereby equipped with an N-terminal $\mathrm{His}_{6}$-SUMO $\operatorname{tag}^{68}$ and a C-terminal EYFP tag, attached via a short linker (DSAGSAGSAG). For interaction studies in bacterial lysate, the AtPIF3/6 genes were subcloned onto a pET- $28 \mathrm{c}$ vector (Novagen) under the control of a T7-lacO promoter, again with C-terminal linkers and EYFP. Variants of the AtPIF proteins were generated in both plasmid contexts, and the identity of all constructs was confirmed by Sanger DNA sequencing (GATC, Konstanz, Germany or Microsynth Seqlab, Göttingen, Germany).

For AtPhyB expression, the plasmid pDG282 was transformed into the E. coli BL21(DE3) strain. Transformant cells were grown in $2 \times 1000 \mathrm{~mL}$ terrific-broth (TB) medium, supplemented with $100 \mu \mathrm{g} \mathrm{mL} \mathrm{m}^{-1}$ streptomycin, at $37^{\circ} \mathrm{C}$ in darkness until an optical density at $600 \mathrm{~nm}\left(\mathrm{OD}_{600}\right)$ of $0.6-0.8$ was reached. $\delta$-Aminolevulinic acid was added at $0.5 \mathrm{mM}$ to assist chromophore production ${ }^{69}$, and the expression was induced by adding $1 \mathrm{mM} \beta$-D-1-thiogalactopyranoside (IPTG). Cultivation continued overnight at $18{ }^{\circ} \mathrm{C}$, before cells were harvested by centrifugation, resuspended in lysis buffer [50 $\mathrm{mM}$ Tris/ $\mathrm{HCl} \mathrm{pH} 8.0,20 \mathrm{mM} \mathrm{NaCl}, 20 \mathrm{mM}$ imidazole; supplemented with protease inhibitor mix (cOmplete Ultra, Roche Diagnostics, Mannheim, Germany)], and lysed by sonification. The cleared lysate was purified by immobilized ion affinity chromatography (IMAC) on Protino NiNTA $1 \mathrm{~mL}$ columns (Macherey-Nagel, Düren, Germany) and eluted with a linear imidazole gradient from 20 to $500 \mathrm{mM}$. Elution fractions were analyzed by denaturing polyacrylamide gel electrophoresis (PAGE), where $1 \mathrm{mM} \mathrm{Zn}^{2+}$ was added to enable detection of covalently incorporated bilin chromophores via zincinduced fluorescence ${ }^{70}$. Suitable fractions were pooled and dialyzed overnight into AEX buffer (20 mM Tris/ $\mathrm{HCl} \mathrm{pH} 8.0,50 \mathrm{mM} \mathrm{NaCl}, 5 \mathrm{mM}$ 2-mercaptoethanol), applied to a HiTrap Q HP $1 \mathrm{~mL}$ anion-exchange column (GE Healthcare Europe $\mathrm{GmbH}$, Freiburg, Germany), and eluted using two successive linear gradients from 50 to $300 \mathrm{mM} \mathrm{NaCl}$ and from 300 to $500 \mathrm{mM}$. Eluted fractions were analyzed by PAGE, appropriately pooled, dialyzed against storage buffer $[10 \mathrm{mM} \mathrm{Tris/HCl} \mathrm{pH8,}$ $10 \mathrm{mM} \mathrm{NaCl}, 10 \%(\mathrm{w} / \mathrm{v})$ glycerol], and stored at $-80^{\circ} \mathrm{C}$.

Purification of the AtPIF3/6-EYFP variants employed a similar protocol with the following differences. No $\delta$-aminolevulinic acid was added, and incubation after induction continued at $16^{\circ} \mathrm{C}$ for $40 \mathrm{~h}$. Following IMAC, the N-terminal $\mathrm{His}_{6^{-}}$ SUMO was cleaved overnight at $4{ }^{\circ} \mathrm{C}$ during dialysis into $50 \mathrm{mM}$ Tris/ $\mathrm{HCl} \mathrm{pH} 8.0$ and $20 \mathrm{mM} \mathrm{NaCl}$ using SENP2-protease. The $\mathrm{His}_{6}$-SUMO tag was removed by IMAC, and the flow-through containing the AtPIF3/6 construct was collected and analyzed by PAGE. Depending upon purity, the proteins were optionally further purified by anion-exchange chromatography as described above. Pure AtPIF3/6EYFP variants were dialyzed into storage buffer and stored at $-80{ }^{\circ} \mathrm{C}$. An analysis by denaturing PAGE of the purified AtPIF3/6-EYFP constructs and the AtPhyB PCM is shown as Supplementary Fig. 9.

Spectroscopic analysis. The concentration of purified AtPhyB PCM and the AtPIF3/6-EYFP variants were determined at $22^{\circ} \mathrm{C}$ by absorption measurements on an Agilent $8453 \mathrm{UV}$-visible spectrophotometer (Agilent Technologies, Waldbronn, Germany). In case of the AtPIF3/6-EYFP variants, a molar extinction coefficient at $513 \mathrm{~nm}$ of $84,300 \mathrm{M}^{-1} \mathrm{~cm}^{-1}$ was used $\mathrm{d}^{71}$. Photoreversible
$\operatorname{Pr} \leftrightarrow \operatorname{Pfr}$ conversion of AtPhyB PCM was ascertained by illumination with lightemitting diodes (LED) with emission wavelengths of $650 \pm 15 \mathrm{~nm}\left(5.6 \mu \mathrm{W} \mathrm{cm}{ }^{-2}\right)$ and $720 \pm 15 \mathrm{~nm}\left(0.7 \mu \mathrm{W} \mathrm{cm}{ }^{-2}\right)$, respectively. Spectra recorded after illumination revealed isosbestic points at 374 and $672 \mathrm{~nm}$. Absorption spectra were also recorded after denaturation in $6.5 \mathrm{M}$ guanidinium hydrochloride. By referencing to the previously reported extinction coefficient for PCB under these conditions $^{72}$, we calculated an extinction coefficient at the isosbestic point $672 \mathrm{~nm}$ for AtPhyB PCM in its native state of $47,600 \mathrm{M}^{-1} \mathrm{~cm}^{-1}$. The fraction of AtPhyB PCM in the Pfr state upon saturating red-light illumination $(640 \mathrm{~nm})$ was determined as described in ref. ${ }^{42}$.

Interaction assay in bacterial lysate. pET-28c plasmids harboring AtPIF3-EYFP or AtPIF6-EYFP variants were transformed into chemically competent BL21(DE3) cells. Three replicate clones were used to inoculate $3 \times 5 \mathrm{~mL}$ TB medium supplemented with $50 \mu \mathrm{g} \mathrm{mL}^{-1}$ kanamycin. Cultures were incubated at $37^{\circ} \mathrm{C}$ up to an $\mathrm{OD}_{600}$ of $0.6-0.8$, at which point temperature was lowered to $16^{\circ} \mathrm{C}$ and expression was induced by addition of $1 \mathrm{mM}$ IPTG. Incubation continued overnight, and cells were harvested by centrifugation at $3000 \times g$ for $10 \mathrm{~min}$. Pelleted cells were resuspended in $300 \mu \mathrm{L}$ lysis buffer [1× FastBreak Cell Lysis Reagent (Promega $\mathrm{GmbH}$, Mannheim, Germany), $10 \mu \mathrm{g} \mathrm{mL} \mathrm{m}^{-1}$ DNaseI (PanReac AppliChem, Darmstadt, Germany), $200 \mu \mathrm{g} \mathrm{mL}{ }^{-1}$ lysozyme (Sigma-Aldrich, Darmstadt, Germany)] and rotated at $22^{\circ} \mathrm{C}$ for $10 \mathrm{~min}$. Cell debris was removed by centrifugation at $186,000 \times g$ for $45 \mathrm{~min}$ using an Optima MAX-XP Ultracentrifuge (BeckmanCoulter, Krefeld, Germany). The concentration of a given AtPIF3/6-EYFP variant in the lysate was determined by absorption measurements at $513 \mathrm{~nm}$ using a CLARIOstar microtiter plate reader (MTP) (BMG Labtech, Ortenberg, Germany). AtPhyB PCM at $2.5 \mu \mathrm{M}$ concentration was mixed with a threefold molar excess of the AtPIF3/6-EYFP variants in 384-well clear MTPs (Thermo Fisher Scientific, Waltham, USA). After illumination with red light $\left(650 \pm 15 \mathrm{~nm}, 5.6 \mu \mathrm{W} \mathrm{cm}{ }^{-2}\right)$ for $4 \mathrm{~min}$, the MTPs were covered with a clear lid, and absorption at 720 and $850 \mathrm{~nm}$ was measured every $5 \mathrm{~min}$ at $28^{\circ} \mathrm{C}$ in an Infinite M200 PRO plate reader (Tecan, Männedorf, Switzerland) for $12 \mathrm{~h}$. After background correction, data at $720 \mathrm{~nm}$ were normalized to the signal of the L-EYFP (Supplementary Table 1) negative control, and the relative initial velocity was determined over the data acquired during the first $4 \mathrm{~h}$

Interaction assays with purified components. Size-exclusion chromatography: The light-dependent interaction between AtPhyB PCM and the AtPIF3/6-EYFP variants was assessed by gel filtration chromatography using a Superdex 200 Increase 10/300 GL (GE Healthcare) column on an ÄKTApure system, equipped with multi-wavelength detection (GE Healthcare). To this end, a mixture of $50 \mu \mathrm{M}$ AtPhyB-PCM and $10 \mu \mathrm{M}$ PIF-EYFP in $67 \mathrm{mM}$ sodium phosphate buffer $\mathrm{pH} 8.0$ and $200 \mathrm{mM} \mathrm{NaCl}$ was prepared and illuminated with 650 - or $720-\mathrm{nm}$ light for 2 min before sample application. Twenty-five microliters of this mixture was applied to the column and separated at a constant $0.75 \mathrm{~mL} \mathrm{~min}^{-1}$ flow rate. Absorption of EYFP and the AtPhyB PCM was measured at 513 and $650 \mathrm{~nm}$, respectively. All proteins were also tested individually, where the AtPIF3/6-EYFP and EYFP samples were not illuminated prior to application.

Fluorescence anisotropy: AtPhyB PCM was illuminated with 640- or 750-nm light for $2 \mathrm{~min}$ immediately prior to the experiment $\left(640 \pm 15 \mathrm{~nm} ; 65 \mu \mathrm{W} \mathrm{cm}{ }^{-2}\right.$ and $750 \pm 15 \mathrm{~nm} ; 420 \mu \mathrm{W} \mathrm{cm}{ }^{-2}$ ). Samples containing $20 \mathrm{nM}$ AtPIF3/6-EYFP and increasing AtPhyB-PCM concentrations between 0 and $10 \mu \mathrm{M}$ were prepared in 20 $\mathrm{mM}$ HEPES/HCl pH 7.3, $10 \mathrm{mM} \mathrm{NaCl}$, and $100 \mu \mathrm{g} \mathrm{mL}^{-1}$ bovine serum albumin, transferred into black 384-well MTPs (Brand, Wertheim, Germany), and illuminated with 640- or 750-nm light. Fluorescence anisotropy of EYFP fluorophore was measured on a CLARIOstar MTP reader (BMG Labtech) with an excitation wavelength of $482 \pm 16 \mathrm{~nm}$, a 504-nm long-pass dichroic filter, and a detection wavelength of $530 \pm 40 \mathrm{~nm}$. The fluorescence gains for the horizontal and vertical detection channels were adjusted to a fluorescence anisotropy value of 0.315, as determined for EYFP with an Olis DSM 172 spectrophotometer (On-Line Instrument Systems, Bogart, USA). Anisotropy data were evaluated with the Fit-omat software ${ }^{73}$ using a single-site binding isotherm:

$$
r=r_{0}+r_{1} \frac{[\mathrm{PhyB}]}{[\mathrm{PhyB}]+K_{\mathrm{D}}}
$$

where $r$ represents the anisotropy of the PIF-EYFP fluorescence, [PhyB] is the concentration of the AtPhyB PCM in either the Pr or Pfr state, and $K_{D}$ is the dissociation constant. For the case of strong binding exhibited by the variants P6.100 and P6, we used a modified single-site binding isotherm that takes into account that the relevant $[\mathrm{PhyB}]$ concentrations are on the same order of magnitude as the constant concentration $c_{\text {total }}$ of the PIF-EYFP protein:

$$
\begin{aligned}
r= & r_{0}+r_{1} / 2 \times\left\{1+[\mathrm{PhyB}] / c_{\text {total }}+K_{\mathrm{D}} / c_{\text {total }}\right. \\
& \left.-\sqrt{\left(1+[\mathrm{PhyB}] / c_{\text {total }}+K_{\mathrm{D}} / c_{\text {total }}\right)^{2}-4[\mathrm{PhyB}] / c_{\text {total }}}\right\}
\end{aligned}
$$

Light-regulated gene expression in mammalian cells. The split transcription factor system for light-controlled gene expression in eukaryotic cells was based on a previously reported set-up ${ }^{36,44}$. To allow ratiometric analysis, this earlier set-up 
was expanded by cloning the Gaussia luciferase under the control of a constitutive promoter onto the same plasmid as the SEAP reporter gene. For testing of the AtPIF variants, AtPIF6 (1-100) was replaced by the corresponding AtPIF1/3/6 derivatives. CHO-K1 (DSMZ, Braunschweig, Germany) were cultivated in HAM's F12 medium (PAN Biotech, Aidenbach, Germany; no. P04-14500) supplemented with $10 \%(\mathrm{v} / \mathrm{v})$ tetracycline-free fetal bovine serum (PAN Biotech; no. P30-3602; batch no. P080317TC) and 1.4\% (v/v) streptomycin (PAN Biotech; no. $\mathrm{P} 06-07100)$. In all, $5 \times 10^{4} \mathrm{CHO}-\mathrm{K} 1$ cells were transfected using polyethyleneimine (PEI; Polysciences Inc. Europe, Hirschberg, Germany; no. 23966-1 ${ }^{74}$. DNA $(0.75 \mu \mathrm{g})$ was diluted in $50 \mu \mathrm{L}$ OptiMEM (Invitrogen, Thermo Fisher Scientific) and mixed with a PEI/OptiMEM mix (2.5 $\mu \mathrm{L}$ PEI solution in $50 \mu \mathrm{L}$ OptiMEM). The DNA-PEI mix was added to the cells after $15 \mathrm{~min}$ of incubation at room temperature. At $4 \mathrm{~h}$ post-transfection, the medium was exchanged. CHO-K1 cells were transfected with the reporter plasmid etr8-CMVmin-SEAP-BGH-SV40-Gaussia (pPF035) and the different AtPhyB:AtPIF variants. All plasmids were transfected in equal amounts (w/w). At $24 \mathrm{~h}$ post-transfection, the cells were supplemented with $15 \mu \mathrm{M}$ phycocyanobilin $(24 \mathrm{mM}$ stock solution in DMSO; Frontier Scientific, Logan, UT, USA; no. P14137) and incubated for $1 \mathrm{~h}$. The cells were then illuminated with $660-\mathrm{nm}$ light for $24 \mathrm{~h}$ at an intensity of $20 \mu \mathrm{mol} \mathrm{m}^{-2} \mathrm{~s}^{-1}$, delivered by custom-built LED light boxes ${ }^{36}$, or kept in darkness. As a negative control, the reporter construct alone was transfected. Photoreversibility was tested by incubating cells for $24 \mathrm{~h}$ under red light, followed by exchange of the media and incubation under far-red light for $24 \mathrm{~h}$. In parallel, cells were incubated in darkness for $48 \mathrm{~h}$ with media exchange after $24 \mathrm{~h}$. Exchange of media and other cell handling was done under $522-\mathrm{nm}$ safe light, to prevent inadvertent actuation of the lightsensitive systems.

SEAP activity assay: The supernatant of transfected cells was transferred to 96well round-bottom MTPs and incubated at $68^{\circ} \mathrm{C}$ for $1 \mathrm{~h}$ to inactivate endogenous phosphatases. Afterwards, $80 \mu \mathrm{L}$ of the supernatant were transferred to 96 -well flatbottom MTPs, and per well $100 \mu \mathrm{L}$ SEAP buffer $[20 \mathrm{mM}$ homoarginine, $1 \mathrm{mM}$ $\mathrm{MgCl}_{2}, 21 \%$ (v/v) diethanolamine] was added ${ }^{36}$. After addition of $20 \mu \mathrm{L} 120 \mathrm{mM}$ para-nitrophenyl phosphate, the absorption at $405 \mathrm{~nm}$ was measured for $1 \mathrm{~h}$ using a BMG Labtech CLARIOstar or a TriStar2 S LB 942 multimode plate reader (Berthold Technologies, Bad Wildbad, Germany) ${ }^{36}$. Outliers were statistically determined and excluded ${ }^{75}$

Gaussia luciferase assay: Twenty microliters of the supernatant of the transfected cells were transferred to a 96-well white MTP and diluted in $60 \mu \mathrm{L}$ phosphate-buffered saline (PBS; $2.68 \mathrm{mM} \mathrm{KCl}, 1.47 \mathrm{mM} \mathrm{KH}_{2} \mathrm{PO}_{4}, 8.03 \mathrm{mM}$ $\mathrm{Na}_{2} \mathrm{PO}_{4}, 137 \mathrm{mM} \mathrm{NaCl}$ ). After addition of $20 \mu \mathrm{L}$ coelenterazine $(472 \mathrm{mM}$ stock solution in methanol, diluted 1:1500 in PBS; Carl Roth, Karlsruhe, Germany, no. 4094.4), the luminescence was measured for 20 min using TriStar2 LB 941 or LB 942 multimode plate readers.

Light-mediated membrane recruitment in mammalian cells. For each AtPIF6 variant tested, a lentiviral vector $(\mathrm{pHR})$ was constructed containing a membranebound AtPhyB PCM (PHY-CAAX, residues 1-650) and a YFP-conjugated AtPIF6 variant. An IRES was introduced between the two coding sequences to ensure regulation of dual expression. Lentivirus was created by transfecting HEK-293T cells with pHR constructs and harvesting filtered media $48 \mathrm{~h}$ post-transfection. Mouse fibroblasts (NIH-3T3) were cultured in Dulbecco's Modified Eagle Medium (DMEM) containing 10\% (v/v) fetal bovine serum. Fibroblasts were treated with lentivirus containing the constructs of interest. For all fibroblast experiments, cells were cultured in a 96-well glass-bottomed plate. Wells were pretreated with fibronectin for $30 \mathrm{~min}$, following which fibronectin was aspirated and cells were plated and spun down for $5 \mathrm{~min}$ at $800 \mathrm{rpm}$. Cells were plated in 96-well glassbottom plates and allowed to adhere for at least $12 \mathrm{~h}$. Imaging was performed using a $\times 60$ oil immersion objective (NA 1.4) on a Nikon TI Eclipse microscope with a CSU-X1 confocal spinning disk, an EM-CCD camera, and appropriate laser lines, dichroics, and filters. DMEM was supplemented with phycocyanobilin 30 min prior to the start of the experiment. Cells were exposed to infrared light followed by red light to cause membrane recruitment and the resulting change in cytoplasmic fluorescence was measured using ImageJ by selecting a cytoplasmic region and computing the average pixel intensity before and after photostimulation. The change in cytoplasmic YFP-PIF level was normalized to the total YFPPIF fluorescence in the nucleus under infrared conditions, to normalize to total expression level differences caused by lentivirus. In these experiments, light was delivered through the microscope using a Mightex Polygon digital micromirror device (DMD), X-Cite XLED1 LED light sources at $635 \pm 20$ and $730 \pm 20 \mathrm{~nm}$, and a $\times 40$ objective lens. The duration of LED illumination was $1 \mathrm{~min}$. To estimate the light dose delivered to the cell, we measured the light intensity using a ThorLabs power meter (PM100D) when the DMD was set to $100 \%$ transmission and obtained $100 \mu \mathrm{W}$ for 635 -nm light and $20 \mu \mathrm{W}$ for 730 -nm light, over a field of view of about $100 \mu \mathrm{m}$ squared. For all experiments, we set the DMDs to $5 \%$ dithering (so each region was only illuminated for $5 \%$ of the time), translating into a final calculated intensity of $5 \mu \mathrm{W} 635-\mathrm{nm}$ light and $1 \mu \mathrm{W}$ of $730-\mathrm{nm}$ light. The light was delivered over an approximately $100 \mu \mathrm{m} \times 100 \mu \mathrm{m}$ field of view, leading to an overall LED power density of $50 \mathrm{~mW} \mathrm{~cm}^{-2}$ at $635 \mathrm{~nm}$ and $10 \mathrm{~mW} \mathrm{~cm}^{-2}$ at $730 \mathrm{~nm}$. Notably, these values are slightly higher but of comparable magnitude to those used by Pathak et al. for the AtPhyB:AtPIF3/6 system in the context of lightregulated gene expression ${ }^{76}$.
Statistics and reproducibility. Data are reported as mean \pm SD or as mean \pm SEM of $n \geq 3$ biologically independent replicates. Details are specified in the legends to the figures and tables. All experiments could be reproduced with similar results.

Reporting summary. Further information on research design is available in the Nature Research Reporting Summary linked to this article.

\section{Data availability}

The data underlying Figs. 2-6 are available in Supplementary Data 1. All data that support the findings of this study are available from the corresponding author upon reasonable request.

Received: 20 July 2019; Accepted: 7 November 2019; Published online: 02 December 2019

\section{References}

1. Butler, W. L., Norris, K. H., Siegelman, H. W. \& Hendricks, S. B. Detection, assay, and preliminary purification of the pigment controlling photoresponsive development of plants. Proc. Natl Acad. Sci. 45, 1703-1708 (1959).

2. Bae, G. \& Choi, G. Decoding of light signals by plant phytochromes and their interacting proteins. Annu. Rev. Plant Biol. 59, 281-311 (2008).

3. Rockwell, N. C. \& Lagarias, J. C. A brief history of phytochromes. Chemphyschem 11, 1172-1180 (2010).

4. Quail, P. H. Phytochrome photosensory signalling networks. Nat. Rev. Mol. Cell Biol. 3, 85-93 (2002).

5. Rockwell, N. C., Su, Y.-S. \& Lagarias, J. C. Phytochrome structure and signaling mechanisms. Annu. Rev. Plant Biol. 57, 837-858 (2006).

6. Takala, H. et al. Signal amplification and transduction in phytochrome photosensors. Nature 509, 245-248 (2014).

7. Burgie, E. S., Zhang, J. \& Vierstra, R. D. Crystal structure of deinococcus phytochrome in the photoactivated state reveals a cascade of structural rearrangements during photoconversion. Structure 24, 448-457 (2016).

8. Song, C. et al. 3D structures of plant phytochrome A as Pr and Pfr from solidstate NMR: implications for molecular function. Front. Plant Sci. 9, 498 (2018).

9. Anders, K., Daminelli-Widany, G., Mroginski, M. A., von Stetten, D. \& Essen, L.-O. Structure of the cyanobacterial phytochrome 2 photosensor implies a tryptophan switch for phytochrome signaling. J. Biol. Chem. 288, 35714-35725 (2013).

10. Assafa, T. E., Anders, K., Linne, U., Essen, L.-O. \& Bordignon, E. Light-driven domain mechanics of a minimal phytochrome photosensory module studied by EPR. Structure 26, 1534-1545.e4 (2018).

11. Gao, R. \& Stock, A. M. Biological insights from structures of two-component proteins. Annu. Rev. Microbiol. 63, 133-154 (2009).

12. Yeh, K. C. \& Lagarias, J. C. Eukaryotic phytochromes: light-regulated serine/ threonine protein kinases with histidine kinase ancestry. Proc. Natl Acad. Sci. USA 95, 13976-13981 (1998).

13. Shin, A.-Y. et al. Evidence that phytochrome functions as a protein kinase in plant light signalling. Nat. Commun. 7, 11545 (2016).

14. Pham, V. N., Kathare, P. K. \& Huq, E. Phytochromes and phytochrome interacting factors. Plant Physiol. 176, 1025-1038 (2018).

15. Casal, J. J. Photoreceptor signaling networks in plant responses to shade. Annu. Rev. Plant Biol. 64, 403-427 (2013).

16. Rausenberger, J. et al. Photoconversion and nuclear trafficking cycles determine phytochrome A's response profile to far-red light. Cell 146, 813-825 (2011).

17. Leivar, P. \& Monte, E. PIFs: systems integrators in plant development. Plant Cell 26, 56-78 (2014).

18. Ni, M., Tepperman, J. M. \& Quail, P. H. PIF3, a phytochrome-interacting factor necessary for normal photoinduced signal transduction, is a novel basic helix-loop-helix protein. Cell 95, 657-667 (1998).

19. Ni, M., Tepperman, J. M. \& Quail, P. H. Binding of phytochrome B to its nuclear signalling partner PIF3 is reversibly induced by light. Nature $\mathbf{4 0 0}$, 781-784 (1999).

20. Martín, G. et al. Phytochrome and retrograde signalling pathways converge to antagonistically regulate a light-induced transcriptional network. Nat. Commun. 7, 11431 (2016)

21. Toledo-Ortiz, G., Huq, E. \& Quail, P. H. The Arabidopsis basic/helix-loophelix transcription factor family. Plant Cell 15, 1749-1770 (2003).

22. Heim, M. A. et al. The basic helix-loop-helix transcription factor family in plants: a genome-wide study of protein structure and functional diversity. Mol. Biol. Evol. 20, 735-747 (2003).

23. Bailey, P. C. et al. Update on the basic helix-loop-helix transcription factor gene family in Arabidopsis thaliana. Plant Cell 15, 2497-2502 (2003). 
24. Khanna, R. et al. A novel molecular recognition motif necessary for targeting photoactivated phytochrome signaling to specific basic helix-loop-helix transcription factors. Plant Cell 16, 3033-3044 (2004).

25. Shimizu-Sato, S., Huq, E., Tepperman, J. M. \& Quail, P. H. A light-switchable gene promoter system. Nat. Biotechnol. 20, 1041-1044 (2002).

26. Matsushita, T., Mochizuki, N. \& Nagatani, A. Dimers of the N-terminal domain of phytochrome B are functional in the nucleus. Nature 424, 571-574 (2003).

27. Levskaya, A., Weiner, O. D., Lim, W. A. \& Voigt, C. A. Spatiotemporal control of cell signalling using a light-switchable protein interaction. Nature 461, 997-1001 (2009).

28. Müller, K. et al. A red/far-red light-responsive bi-stable toggle switch to control gene expression in mammalian cells. Nucleic Acids Res. 41, e77 (2013).

29. Buckley, C. E. et al. Reversible optogenetic control of subcellular protein localization in a live vertebrate embryo. Dev. Cell 36, 117-126 (2016)

30. Yousefi, O. S. et al. Optogenetic control shows that kinetic proofreading regulates the activity of the T cell receptor. eLife 8, e42475 (2019).

31. Huq, E. et al. Phytochrome-interacting factor 1 is a critical bHLH regulator of chlorophyll biosynthesis. Science 305, 1937-1941 (2004).

32. Lee, N. \& Choi, G. Phytochrome-interacting factor from Arabidopsis to liverwort. Curr. Opin. Plant Biol. 35, 54-60 (2017).

33. Deisseroth, K. et al. Next-generation optical technologies for illuminating genetically targeted brain circuits. J. Neurosci. 26, 10380-10386 (2006).

34. Ziegler, T. \& Möglich, A. Photoreceptor engineering. Front. Mol. Biosci. 2, 30 (2015).

35. Müller, K. et al. Multi-chromatic control of mammalian gene expression and signaling. Nucleic Acids Res. 41, e124 (2013).

36. Müller, K., Zurbriggen, M. D. \& Weber, W. Control of gene expression using a red- and far-red light-responsive bi-stable toggle switch. Nat. Protoc. 9, 622-632 (2014).

37. Toettcher, J. E., Gong, D., Lim, W. A. \& Weiner, O. D. Light-based feedback for controlling intracellular signaling dynamics. Nat. Methods 8, 837-839 (2011).

38. Toettcher, J. E., Weiner, O. D. \& Lim, W. A. Using optogenetics to interrogate the dynamic control of signal transmission by the Ras/Erk module. Cell 155, 1422-1434 (2013).

39. Smith, R. W. et al. Interactions between phyB and PIF proteins alter thermal reversion reactions in vitro. Photochem. Photobiol. 93, 1525-1531 (2017).

40. Burgie, E. S., Bussell, A. N., Walker, J. M., Dubiel, K. \& Vierstra, R. D. Crystal structure of the photosensing module from a red/far-red light-absorbing plant phytochrome. Proc. Natl Acad. Sci. USA 111, 10179-10184 (2014).

41. Castillon, A., Shen, H. \& Huq, E. Phytochrome interacting factors: central players in phytochrome-mediated light signaling networks. Trends Plant Sci. 12, 514-521 (2007).

42. Butler, W. L., Hendricks, S. B. \& Siegelman, H. W. Action spectra of phytochrome in vitro. Photochem. Photobiol. 3, 521-528 (1964).

43. Hörner, M. et al. Light-controlled affinity purification of protein complexes exemplified by the resting ZAP70 interactome. Front. Immunol. 10, 226 (2019).

44. Müller, K. et al. A red light-controlled synthetic gene expression switch for plant systems. Mol. Biosyst. 10, 1679-1688 (2014).

45. Leivar, P. et al. The arabidopsis phytochrome-interacting factor PIF7, together with PIF3 and PIF4, regulates responses to prolonged red light by modulating phyB levels. Plant Cell 20, 337-352 (2008).

46. Monte, E. et al. The phytochrome-interacting transcription factor, PIF3, acts early, selectively, and positively in light-induced chloroplast development Proc. Natl Acad. Sci. 101, 16091-16098 (2004).

47. Kim, J. et al. Functional characterization of phytochrome interacting factor 3 in phytochrome-mediated light signal transduction. Plant Cell 15, 2399-2407 (2003).

48. Penfield, S., Josse, E.-M. \& Halliday, K. J. A role for an alternative splice variant of PIF6 in the control of Arabidopsis primary seed dormancy. Plant Mol. Biol. 73, 89-95 (2010).

49. Ni, W. et al. PPKs mediate direct signal transfer from phytochrome photoreceptors to transcription factor PIF3. Nat. Commun. 8, 15236 (2017).

50. Legris, M. et al. Phytochrome B integrates light and temperature signals in Arabidopsis. Science 354, 897-900 (2016).

51. Li, F.-W. et al. Phytochrome diversity in green plants and the origin of canonical plant phytochromes. Nat. Commun. 6, 7852 (2015).

52. Sakuraba, Y. et al. Phytochrome-interacting transcription factors PIF4 and PIF5 induce leaf senescence in Arabidopsis. Nat. Commun. 5, 4636 (2014).

53. Losi, A., Gardner, K. H. \& Möglich, A. Blue-light receptors for optogenetics. Chem. Rev. 118, 10659-10709 (2018).

54. Hörner, M. et al. Phytochrome-based extracellular matrix with reversibly tunable mechanical properties. Adv. Mater. 31, 1806727 (2019).

55. Gasser, C. et al. Engineering of a red-light-activated human cAMP/cGMPspecific phosphodiesterase. Proc. Natl Acad. Sci. USA 111, 8803-8808 (2014).
56. Ryu, M.-H. et al. Engineering adenylate cyclases regulated by near-infrared window light. Proc. Natl Acad. Sci. USA 111, 10167-10172 (2014)

57. Etzl, S., Lindner, R., Nelson, M. D. \& Winkler, A. Structure-guided design and functional characterization of an artificial red light-regulated guanylate/adenylate cyclase for optogenetic applications. J. Biol. Chem. 293, 9078-9089 (2018).

58. Stabel, R. et al. Revisiting and redesigning light-activated cyclicmononucleotide phosphodiesterases. J. Mol. Biol. 431, 3029-3045 (2019).

59. Kaberniuk, A. A., Shemetov, A. A. \& Verkhusha, V. V. A bacterial phytochrome-based optogenetic system controllable with near-infrared light. Nat. Methods 13, 591-597 (2016).

60. Redchuk, T. A., Omelina, E. S., Chernov, K. G. \& Verkhusha, V. V. Nearinfrared optogenetic pair for protein regulation and spectral multiplexing. Nat. Chem. Biol. 13, 633-639 (2017).

61. Shcherbakova, D. M., Shemetov, A. A., Kaberniuk, A. A. \& Verkhusha, V. V. Natural photoreceptors as a source of fluorescent proteins, biosensors, and optogenetic tools. Annu. Rev. Biochem. 84, 519-550 (2015).

62. Horsten, S. et al. Mapping light-driven conformational changes within the photosensory module of plant phytochrome B. Sci. Rep. 6, 34366 (2016)

63. Nagatani, A. Phytochrome: structural basis for its functions. Curr. Opin. Plant Biol. 13, 565-570 (2010).

64. Kikis, E. A., Oka, Y., Hudson, M. E., Nagatani, A. \& Quail, P. H. Residues clustered in the light-sensing knot of phytochrome B are necessary for conformer-specific binding to signaling partner PIF3. PLoS Genet. 5, e1000352 (2009).

65. Gibson, D. G. et al. Enzymatic assembly of DNA molecules up to several hundred kilobases. Nat. Methods 6, 343-345 (2009).

66. Mukougawa, K., Kanamoto, H., Kobayashi, T., Yokota, A. \& Kohchi, T. Metabolic engineering to produce phytochromes with phytochromobilin, phycocyanobilin, or phycoerythrobilin chromophore in Escherichia coli. FEBS Lett. 580, 1333-1338 (2006).

67. Beyer, H. M. et al. AQUA cloning: a versatile and simple enzyme-free cloning approach. PLoS ONE 10, e0137652 (2015).

68. Panavas, T., Sanders, C. \& Butt, T. R. SUMO fusion technology for enhanced protein production in prokaryotic and eukaryotic expression systems. Methods Mol. Biol. 497, 303-317 (2009).

69. Gambetta, G. A. \& Lagarias, J. C. Genetic engineering of phytochrome biosynthesis in bacteria. Proc. Natl Acad. Sci. 98, 10566-10571 (2001).

70. Berkelman, T. R. \& Lagarias, J. C. Visualization of bilin-linked peptides and proteins in polyacrylamide gels. Anal. Biochem. 156, 194-201 (1986).

71. Shaner, N. C., Steinbach, P. A. \& Tsien, R. Y. A guide to choosing fluorescent proteins. Nat. Methods 2, 905-909 (2005).

72. Lamparter, T., Esteban, B. \& Hughes, J. Phytochrome Cph1 from the cyanobacterium Synechocystis PCC6803. Eur. J. Biochem. 268, 4720-4730 (2001)

73. Möglich, A. An open-source, cross-platform resource for nonlinear leastsquares curve fitting. J. Chem. Educ. 95, 2273-2278 (2018).

74. Baaske, J. et al. Dual-controlled optogenetic system for the rapid downregulation of protein levels in mammalian cells. Sci. Rep. 8, 15024 (2018).

75. Jacobs, J. L. \& Dinman, J. D. Systematic analysis of bicistronic reporter assay data. Nucleic Acids Res. 32, e160 (2004).

76. Pathak, G. P., Strickland, D., Vrana, J. D. \& Tucker, C. L. Benchmarking of optical dimerizer systems. ACS Synth. Biol. 3, 832-838 (2014).

\section{Acknowledgements}

We thank members of our laboratories for support and comments; Dr. J. Casal for fruitful discussion; Dr. B. Höcker for chromatography use; Dr. T. Scheibel for ultracentrifuge use and Dr. M. Hörner and Dr. W. Weber for generously providing PhyB expression plasmids. Funding through the Deutsche Forschungsgemeinschaft (grants MO2192/7-1 to A.M., ZU259/2-1 to M.D.Z., and ES152/16 to L.-O.E.), under Germany's Excellence Strategy CEPLAS EXC2048/1 (to M.D.Z., ID 390686111), by NIH grant DP2EB024247 (to J.E.T.), by NIH training grant T32GM007388 (to S.G.J.), and by the European Commission - Research Executive Agency (H2020 Future and Emerging Technologies (FETOpen) Project ID 801041 CyGenTiG to M.D.Z.) is appreciated. J.R.W.K. gratefully acknowledges support through the ENB program "Biological Physics."

\section{Author contributions}

D.G. designed and cloned the AtPIF variants, expressed them and AtPhyB, analyzed light-dependent interactions in lysate and for purified proteins, cloned the constructs for light-regulated gene expression, and analyzed data. P.F. performed and evaluated experiments on light-regulated gene expression. S.G.J. conducted and evaluated experiments on light-regulated membrane recruitment. J.R.W.K. cloned and expressed several AtPIF variants and analyzed their interactions with AtPhyB in lysate. L.-O.E. advised on experimental design. J.E.T. supervised experiments on light-regulated membrane recruitment. M.D.Z. conceived the project and designed and supervised research. A.M. conceived the project and designed and supervised research. D.G., P.F., M.D.Z., and A.M. wrote the manuscript with input from all authors. 


\section{Competing interests}

The authors declare no competing interests.

\section{Additional information}

Supplementary information is available for this paper at https://doi.org/10.1038/s42003019-0687-9.

Correspondence and requests for materials should be addressed to M.D.Z. or A.M.

Reprints and permission information is available at http://www.nature.com/reprints

Publisher's note Springer Nature remains neutral with regard to jurisdictional claims in published maps and institutional affiliations. (c) (i) Open Access This article is licensed under a Creative Commons Attribution 4.0 International License, which permits use, sharing, adaptation, distribution and reproduction in any medium or format, as long as you give appropriate credit to the original author(s) and the source, provide a link to the Creative Commons license, and indicate if changes were made. The images or other third party material in this article are included in the article's Creative Commons license, unless indicated otherwise in a credit line to the material. If material is not included in the article's Creative Commons license and your intended use is not permitted by statutory regulation or exceeds the permitted use, you will need to obtain permission directly from the copyright holder. To view a copy of this license, visit http://creativecommons.org/ licenses/by/4.0/.

(C) The Author(s) 2019 